\title{
Enhancing the expressive power of Sugeno integrals for qualitative data analysis
}

\author{
Miguel Couceiro ${ }^{1}$, Didier Dubois ${ }^{2}$, Henri Prade $^{2}$, and Agnès Rico ${ }^{3}$ \\ 1 LORIA, Nancy, France \\ miguel.couceiro@inria.fr \\ 2 IRIT-CNRS, Toulouse, France \\ \{dubois, prade\}@irit.fr \\ 3 ERIC, Université de Lyon, France \\ agnes.rico@univ-lyon1.fr
}

\begin{abstract}
Sugeno integrals are useful for describing families of multiple criteria aggregation functions qualitatively. It is known that Sugeno integrals, as aggregation functions, can be represented by a set of rules. Each rule refers to the same threshold in the conditions about the values of the criteria and in the conclusion pertaining to the value of the integral. However, in the general case we expect rules where several thresholds appear. Some of these rules involving different thresholds can be represented by Sugeno utility functionals where criteria values are rescaled by means of utility functions associated with each criterion. But as shown in this paper, their representation power is quite restrictive. In contrast, we provide evidence to conjecture that the use of disjunctions or conjunctions of Sugeno integrals with utility functions drastically improves the expressive power and that they can capture any aggregation function on a finite scale, understood as piecewise unary aggregation functions.
\end{abstract}

Keywords: Sugeno integrals; piecewise unary functions; rule-based representation.

\section{Introduction}

Sugeno integrals are aggregation functions that return a global evaluation in-between the minimum and the maximum of the combined partial evaluations. They are used in multiple criteria decision making and in decision under uncertainty $[10,6,9,16]$. They are qualitative aggregation functions because they can be defined on any completely ordered scale. The idea is to use a lattice polynomial (using min and max operations) whereby the importance of each subset of criteria is assessed by means of a monotonic set-function called a capacity. Sugeno integrals include weighted minimum and weighted maximum as particular cases.

The problem of representing a function of several variables with a Sugeno integral is discussed in [16]. More precisely, for a given piece of data composed of a vector of partial evaluations and a global evaluation, the set of Sugeno integrals that agree with this piece of data is determined. Moreover, necessary and sufficient conditions are presented.

The problem of eliciting Sugeno integrals agreeing with a set of data has received some attention both from a theoretical and a practical point of view [15, 14]. The idea is to define a pair of best upper and lower capacities with importance weights bearing on the same subsets of criteria, corresponding to a pair of Sugeno integrals that enclose the dataset. For each piece of data, this approach computes tightest constraints from above and constraints from below on the capacity needed for representing the dataset. In [14], a general approach to the elicitation of several such families of Sugeno integrals 
is proposed in cases in which the data are not altogether compatible with a unique family of capacities.

In $[11,8]$ a Sugeno integral $S$ is shown to represent a set of single-threshold if-then rules of the form $x \geq \alpha$ and $y \geq \alpha$ and $\ldots z \geq \alpha \Rightarrow S \geq \alpha$, or yet, $x \leq \alpha$ and $y \leq \alpha$ and $\ldots z \leq \alpha \Rightarrow S \leq \alpha$. These representations are used to select or reject some alternatives, respectively.

Recently, Sugeno integral has been generalized into Sugeno utility functionals [3] that introduce a utility function for each criterion. In the domain of multiple criteria decision making, this aggregation function can be viewed as the combination of the Sugeno integral and order preserving one-argument maps on each criterion. In [4] the Sugeno utility functional is extended to distributive lattices with more general maps.

In this paper, we take a step beyond the above results by considering disjunctions or conjunctions of Sugeno utility functionals. We claim that this class covers all monotonic piecewise unary functions on finite scales, and can represent multi-threshold rules of the form

$$
x \geq \alpha \text { and } y \geq \beta \text { and } \ldots z \geq \gamma \Rightarrow S \geq \delta \text { (selection rules); }
$$

or yet

$$
x \leq \alpha \text { and } y \leq \beta \text { and } \ldots z \leq \gamma \Rightarrow S \leq \delta \text { (deletion rules); }
$$

The paper is organized as follows: The next section is devoted to the background on Sugeno integrals and the kind of if-then rules they can represent. Section 3 presents necessary and sufficient conditions for a set of rules to be represented with a Sugeno utility functional, that is Sugeno integral on utility functions that modify the value scale of each criterion. The main purpose of Section 4 is the extension of Sugeno utility functionals to conjunctive and disjunctive combinations thereof, that capture the class of non-decreasing piecewise unary functions. This class of functions is shown to be very expressive and can capture any aggregation function on a finite scale.

\section{Sugeno integrals and qualitative datasets}

We use the terminology of multiple criteria decision-making where some objects are evaluated according to criteria. We denote by $C=\{1, \cdots, n\}$ the set of criteria, $2^{C}$ the power set and $L$ a totally ordered scale with top 1 , bottom 0 , and the order-reversing operation denoted by $\nu$ ( $\nu$ is involutive and such that $\nu(1)=0$ and $\nu(0)=1)$. An object is represented by a vector $x=\left(x_{1}, \ldots, x_{n}\right)$ where $x_{i}$ is the evaluation of $x$ according to criterion $i$.

Sugeno integral In the definition of Sugeno integral the relative weights of the set of criteria are represented by a capacity (or fuzzy measure) which is a set function $\mu: 2^{C} \rightarrow L$ that satisfies $\mu(\emptyset)=0, \mu(C)=1$ and $A \subseteq B$ implies $\mu(A) \leq \mu(B)$. The conjugate capacity of $\mu$ is defined by $\mu^{c}(A)=\nu\left(\mu\left(A^{c}\right)\right)$ where $A^{c}$ is the complement of $A$. Sugeno integral was originally defined in [17, 18]. The most common definition is as follows:

Definition 1. The Sugeno integral of a function $x: i \in C \mapsto x_{i} \in L$ with respect to a capacity $\mu: 2^{C} \rightarrow L$ is defined by:

$S_{\mu}(x)=\max _{\alpha \in L} \min (\alpha, \mu(x \geq \alpha))$, where $\mu(x \geq \alpha)=\mu\left(\left\{i \in C \mid x_{i} \geq \alpha\right\}\right)$.

It can be equivalently written under various forms $[17,7,13,12]$, especially:

$$
S_{\mu}(x)=\max _{A \subseteq \mathcal{C}} \min \left(\mu(A), \min _{i \in A} x_{i}\right)=\min _{A \subseteq \mathcal{C}} \max \left(\mu\left(A^{c}\right), \max _{i \in A} x_{i}\right)
$$


Sugeno integrals compatible with a dataset Let us recall how to elicit a family of Sugeno integrals that are compatible with a given dataset that is a collection of pairs $\left(x^{k}, \alpha_{k}\right), k=1, \ldots, N$ where each $x^{k}$ is a tuple $\left(x_{1}^{k}, \ldots, x_{n}^{k}\right)$ of local evaluations of object $k$ with respect to criteria $i \in C$ and $\alpha_{k}$ is the global evaluation of object $k$.

In [16] it is proved that for a given piece of data $(x, \alpha)$ the set of capacities $\mu$ such that $S_{\mu}(x)=\alpha$ is such that $\forall A \subseteq C, \check{\mu}_{x, \alpha}(A) \leq \mu(A) \leq \hat{\mu}_{x, \alpha}(A)$, where $\check{\mu}_{x, \alpha}$ and $\hat{\mu}_{x, \alpha}$ are capacities defined by

$$
\check{\mu}_{x, \alpha}(A)=\left\{\begin{array}{l}
\alpha \text { if }\left\{i \mid x_{i} \geq \alpha\right\} \subseteq A \\
0 \text { otherwise }
\end{array} \text { and } \hat{\mu}_{x, \alpha}(A)=\left\{\begin{array}{l}
\alpha \text { if } A \subseteq\left\{i \mid x_{i}>\alpha\right\} \\
1 \text { otherwise }
\end{array}\right.\right.
$$

Note that $\check{\mu}_{x, \alpha}$ is a necessity measure with respect to the possibility distribution

$$
\check{\pi}_{x, \alpha}(i)=\left\{\begin{array}{l}
1 \text { if } x_{i} \geq \alpha \\
\nu(\alpha) \text { otherwise }
\end{array},\right.
$$

and $\hat{\mu}_{x, \alpha}(A)$ is a possibility measure with respect to the possibility distribution

$$
\hat{\pi}_{x, \alpha}(i)=\left\{\begin{array}{l}
1 \text { if } x_{i} \leq \alpha \\
\alpha \text { otherwise }
\end{array} .\right.
$$

It is worth noticing that a capacity $\mu$ is compatible with the piece of data $(x, \alpha)$ in the above sense if and only if $\mu(x>\alpha) \leq \alpha$ and $\mu(x \geq \alpha) \geq \alpha$. Note that for the set of compatible $\mu$ 's to be not empty we need that $\min _{i=1}^{n} x_{i} \leq \alpha \leq \max _{i=1}^{n} x_{i}$, due to idempotence.

The set of capacities compatible with the dataset $\left(x^{k}, \alpha_{k}\right)_{k}$ is the set of capacities $\mu$ satisfying $\max _{k} \breve{\mu}_{x^{k}, \alpha_{k}} \leq \mu \leq \min _{k} \hat{\mu}_{x^{k}, \alpha_{k}}$. This set of solutions can be empty, even if the set of compatible $\mu$ 's is not empty for each piece of data. In order to compare $\max _{k} \breve{\mu}_{x^{k}, \alpha_{k}}$ and $\min _{k} \hat{\mu}_{x^{k}, \alpha_{k}}$ it is not necessary to calculate their values and to compare them on each subset of criteria. It is proved in [16] that the set of compatible capacities is not empty if and only if for all $\alpha_{k}<\alpha_{l}$ we have $\left\{i \mid x_{i}^{l} \geq \alpha_{l}\right\} \nsubseteq\left\{i \mid x_{i}^{k}>\alpha_{k}\right\}$.

Sugeno integral as a set of if-then rules In [14] it is described how to express if-then rules associated to Sugeno integrals. We have two sorts of rules: selection rules and deletion rules. Their construction is based on the inner qualitative Moebius transform of a capacity $\mu$ which is a mapping $\mu_{\#}: 2^{C} \rightarrow L$ defined by

$$
\mu_{\#}(E)=\mu(E) \text { if } \mu(E)>\max _{B \subset E} \mu(B) \text { and } 0 \text { otherwise. }
$$

A set $E$ such that $\mu_{\#}(E)>0$ is called a focal set. The set of focal sets of $\mu$ is denoted by $\mathcal{F}(\mu)$. Sugeno integral can be expressed in terms of $\mu_{\#}$ using Equation (1) as follows [8]:

$$
S_{\mu}(x)=\max _{E \in \mathcal{F}(\mu)} \min \left(\mu_{\#}(E), \min _{i \in E} x_{i}\right)=\min _{T \in \mathcal{F}\left(\mu^{c}\right)} \max \left(\nu\left(\mu_{\#}^{c}(T)\right), \max _{i \in T} x_{i}\right) .
$$

A selection rule is a rule whose conclusion is of the form $S \geq \alpha$. A deletion rule is a rule whose conclusion is of the form $S \leq \alpha$. A Sugeno integral corresponds to the following rules:

- Selection rules associated to $S_{\mu}$ Each focal set $E$ of $\mu$ corresponds to the selection rule: 


$$
R_{E}^{s} \text { : If } x_{i} \geq \mu_{\#}(E) \text { for all } i \in E \text { then } S_{\mu}(x) \geq \mu_{\#}(E) .
$$

- Deletion rules associated to $S_{\mu}$ Each focal set $T$ of the conjugate $\mu^{c}$ corresponds to the deletion rule:

$$
R_{T}^{e} \text { : If } x_{i} \leq \nu\left(\mu_{\#}^{c}(T)\right) \text { for all } i \in T \text { then } S_{\mu}(x) \leq \nu\left(\mu_{\#}^{c}(T)\right) .
$$

Note that a Sugeno integral is equivalent to a set of single-thresholded rules. In the following, single-thresholded selection rules will be denoted by $\left(\bigwedge_{i \in E_{j}} x_{i} \geq \delta_{j}\right) \Rightarrow S(x) \geq \delta_{j}$ and singlethresholded deletion rules will be denoted by $\left(\bigwedge_{i \in T_{j}} x_{i} \leq \delta_{j}\right) \Rightarrow S(x) \leq \delta_{j}$.

As Sugeno integrals are idempotent, the set of selection rules of the form $\left(\bigwedge_{i \in C} x_{i} \geq \delta_{j}\right) \Rightarrow$ $S(x) \geq \delta_{j}$ or deletion rules of the form $\left(\bigwedge_{i \in C} x_{i} \leq \delta_{j}\right) \Rightarrow S(x) \leq \delta_{j}$, is always valid.

Let us denote by $r_{i}, i \in I$ the rules in a single-thresholded rule set $R$, and $A^{r_{i}} i \in I$ the set of criteria involved in the rule $r_{i}$ and $\delta_{i} i \in I$ the associated threshold. In some cases, we can define a capacity $\mu$ with focal sets $A^{r_{i}}$ such that $\mu_{\#}\left(A^{r_{i}}\right)=\delta_{i}$ such that the corresponding Sugeno integral induces $R$.

Proposition 1. A set of single-thresholded selection rules $R$ is representable by a Sugeno integral provided that

- if $A^{r_{1}} \subset A^{r_{2}}$ then $\delta_{1}<\delta_{2}$

- if $A^{r_{1}}=A^{r_{2}} \neq C$ then $\delta_{1}=\delta_{2}$.

Proof We define the capacity induced by $R$ as above. The first condition is due to the fact that two focal sets cannot have the same weight if one is a subset of the other. Moreover the second condition reflects the unicity of the weight of a focal set (if $A^{r_{1}}=A^{r_{2}}=C$, any $\delta_{1}, \delta_{2}$ are possible due to idempotence).

This result indicates in particular that if we wish to represent the pair of rules $x_{1} \geq \alpha$ and $x_{2} \geq \alpha \Rightarrow S \geq \alpha$ and $x_{1} \geq \beta$ and $x_{2} \geq \beta \Rightarrow S \geq \beta$, where $\{1,2\} \subset C$ (there are more than two criteria), then we need to go beyond pure Sugeno integrals: we need at least two of them.

\section{Generalizing Sugeno integrals with utility functions}

In this paper we are going to consider multi-thresholded rules. It is then clear we need to go beyond the mere use of Sugeno integrals. A first generalization is the following:

Definition 2. The Sugeno utility functional with respect to a capacity $\mu$ is $S_{\mu, \varphi}(x)=S_{\mu}(\varphi(x))$ where $\varphi(x)=\left(\varphi_{1}\left(x_{1}\right), \cdots, \varphi_{n}\left(x_{n}\right)\right)$ and each mapping $\varphi_{i}: L \rightarrow L$ is an increasing function in the wide sense, with limit conditions $\varphi_{i}(0)=0$ and $\varphi_{i}(1)=1$.

Note that $S_{\mu, \varphi}(x)=\max _{E \in \mathcal{F}(\mu)} \min \left(\mu_{\#}(E), \min _{i \in E} \varphi_{i}\left(x_{i}\right)\right)$. It is also worth noticing that $S_{\mu, \varphi}$ is not always an idempotent aggregation function. Note that when the value scale $L$ is finite, the effect of function $\varphi_{i}$ is essentially one of shrinking the value scale since when $\varphi_{i}$ is not the identity, $\varphi_{i}(L) \subset L$. Despite this remark, Sugeno utility functionals are strictly more expressive than Sugeno integrals, as shown in [2], for instance.

It is easy to figure out that $S_{\mu, \varphi}$ expresses the rules:

$$
\left(\bigwedge_{i \in E_{j}} \varphi_{i}\left(x_{i}\right) \geq \delta_{j}\right) \Rightarrow S_{\mu, \varphi}(x) \geq \delta_{j} \text { and }\left(\bigwedge_{i \in T_{j}} \varphi_{i}\left(x_{i}\right) \leq \delta_{j}\right) \Rightarrow S_{\mu, \varphi}(x) \leq \delta_{j} .
$$


Let $\alpha_{i}$ be such that $\varphi_{i}\left(\alpha_{i}\right)=\delta_{j}$. Then the above single-thresholded rules express multi-thresholded selection rules of the form $\left(\bigwedge_{i \in E_{j}} x_{i} \geq \alpha_{i}\right) \Rightarrow S_{\mu, \varphi}(x) \geq \delta_{j}$. Let us show that any multi-thresholded selection rule can be represented by a Sugeno utility functional.

Example 1. Consider the selection rule $x_{1} \geq \alpha$ and $x_{2} \geq \beta \Rightarrow S \geq \delta$ with $1 \geq \alpha \geq \beta>\delta \geq 0$, where $C$ contains at least 3 criteria. Define utility functions $\varphi_{1}, \varphi_{2}$ such that $\varphi_{1}\left(x_{1}\right) \geq \delta$ if $x_{1} \geq \alpha$ and $\varphi_{1}\left(x_{1}\right)<\delta$ otherwise; $\varphi_{2}\left(x_{2}\right) \geq \delta$ if $x_{2} \geq \beta$ and $\varphi_{2}\left(x_{2}\right)<\delta$ otherwise. Then we do have that the single-thresholded rule $\varphi_{1}\left(x_{1}\right) \geq \delta$ and $\varphi_{2}\left(x_{2}\right) \geq \delta \Rightarrow S \geq \delta$ is equivalent to the previous multi-thresholded rule. This is because, by construction, $x_{1} \geq \alpha$ is equivalent to $\varphi_{1}\left(x_{1}\right) \geq \delta$, and likewise for $x_{2}$. Then we can use a capacity with weight $\delta$ assigned to focal set $\{1,2\}$ and weight 1 assigned to $C$; the Sugeno utility functional $\max \left(\min \left(\varphi_{1}\left(x_{1}\right), \varphi_{2}\left(x_{2}\right), \delta\right), \min _{i \in C} \varphi_{i}\left(x_{i}\right)\right)$ induces the original multi-thresholded selection rule, provided that, for $i>2$ we let $\varphi_{i}(1)=1$ and $\varphi_{i}\left(x_{i}\right)=0$ otherwise.

More generally, consider the selection rule $x_{1} \geq \alpha_{1}$ and $x_{2} \geq \alpha_{2}$ and $\ldots x_{\ell} \geq \alpha_{\ell} \Rightarrow S \geq \lambda_{j}$, with weights on a finite scale $L=\left\{0, \lambda_{1}<\cdots<\lambda_{k}=1\right\}$. We can represent its effect by means of utility functions $\varphi_{i}$ such that $\varphi_{i}\left(x_{i}\right) \in\left[\lambda_{j}, 1\right]$ if $x_{i} \geq \alpha_{i}$ and $\varphi_{i}\left(x_{i}\right) \in\left[0, \lambda_{j-1}\right]$ otherwise. The weight $\lambda_{j}$ assigned to focal set $\{1,2, \ldots, \ell\}$ and weight 1 assigned to $C$; the Sugeno utility functional representing the rule is $\max \left(\min \left(\min _{i=1}^{\ell} \varphi_{i}\left(x_{i}\right), \lambda_{j}\right), \min _{i \in C} \varphi_{i}\left(x_{i}\right)\right)$, provided that, for $i>\ell$ we let $\varphi_{i}(1)=1$ and $\varphi_{i}\left(x_{i}\right)=0$ otherwise.

Likewise, for the deletion rule, $x_{1} \leq \alpha_{1}$ and $x_{2} \leq \alpha_{2}$ and $\ldots x_{\ell} \leq \alpha_{\ell} \Rightarrow S \leq \lambda_{j}$, we can represent its effect by means of utility functions $\psi_{i}$ such that $\psi_{i}\left(x_{i}\right) \in\left[0, \lambda_{j}\right]$ if $x_{i} \leq \alpha_{i}$ and $\psi_{i}\left(x_{i}\right) \in\left[\lambda_{j+1}, 1\right]$ otherwise. The weight $\lambda_{j}$ assigned to focal set $\{1,2, \ldots, \ell\}$ and weight 1 assigned to $C$; the Sugeno utility functional representing the rule is $S_{\mu, \psi}(x)=\min \left(\max \left(\max _{i=1}^{\ell} \psi_{i}\left(x_{i}\right), \lambda_{j}\right), \max _{i \in C} \psi_{i}\left(x_{i}\right)\right)$, provided that, for $i>\ell$ we let $\psi_{i}(0)=0$ and $\psi_{i}\left(x_{i}\right)=1$ otherwise.

However, it is sometimes impossible to represent the behavior of several rules by a single Sugeno utility functional, because the constraints on the utility functions induced by the rules may be in conflict.

Proposition 2. Consider two selection rules $r^{1}$ and $r^{2}$ sharing one criterion $x$, and of the form "if ... and $x \geq \alpha_{i}$ and ... then $S \geq \delta_{i}$ such that $\alpha_{1}>\alpha_{2}$ but $\delta_{1} \leq \delta_{2}$. There is no Sugeno integral with utility functions that can represent both of them.

Proof: Indeed the utility function $\varphi$ for criterion $x$ is submitted to the following constraints: for rule 1: $\varphi(x) \geq \delta_{1}$ if $x \geq \alpha_{1}$, and $\varphi(x)<\delta_{1}$ otherwise. For rule $2: \varphi(x) \geq \delta_{2}$ if $x \geq \alpha_{2}$, and $\varphi(x)<\delta_{2}$ otherwise. But since $\alpha_{1}>\alpha_{2}$, suppose $\alpha_{1}>x \geq \alpha_{2}$. Then the conditions enforce $\varphi(x)<\delta_{1}$ and $\varphi(x) \geq \delta_{2}$, which is impossible.

Example 2. Let us consider the rules: $\left\{\begin{array}{l}\text { if } x_{1} \geq \lambda_{2} \text { and } x_{2} \geq \lambda_{3} \text { then } S \geq \lambda_{3} \\ \text { if } x_{2} \geq \lambda_{2} \text { and } x_{3} \geq \lambda_{2} \text { then } S \geq \lambda_{3}\end{array}\right\}$ where $\lambda_{2}<\lambda_{3}$. Variable $x_{2}$ is common to both rules. Due to the first rule, we must add a utility function $\varphi_{2}$ such that $\varphi_{2}\left(\lambda_{3}\right) \geq \lambda_{3}$ and $\varphi_{2}\left(x_{2}\right)<\lambda_{3}$ if $x_{2}<\lambda_{3}$ (for instance $\varphi_{2}\left(x_{2}\right)=x_{2}$ ). In particular, $\varphi_{2}\left(\lambda_{2}\right)<\lambda_{3}$. But according to the other rule, one must have that $\varphi_{2}\left(\lambda_{2}\right) \geq \lambda_{3}$, which creates a contradiction.

Note that in the above proposition, if the two rules involve the same criteria $\left(A^{r_{1}}=A^{r_{2}}\right)$ and the thresholds in $r^{1}$ for criteria other than $x$ are not less than the thresholds in $r^{2}$ for these criteria, then rule $r^{1}$ is just a consequence of rule $r^{2}$ and can be dropped.

A proposition similar to Proposition 2 would hold for deletion rules. 
If the condition in the above proposition is not encountered in a set of selection rules $R$, that is, $\forall r_{1}, r_{2} \in R, \forall i \in A^{r_{1}} \cap A^{r_{2}}$, if $\alpha_{i}^{r_{1}}>\alpha_{i}^{r_{2}}$ implies $\delta_{1}>\delta_{2}$, then the set of rules can be accounted for by a Sugeno integral based on a capacity $\mu$ such that $\mu\left(A^{r_{j}}\right)=\delta_{j}, r^{j} \in R$, provided that we delete redundant rules from $R$.

Example 3. Let us consider the rules: $\left\{\begin{array}{l}\text { if } x_{1} \geq \lambda_{3} \text { and } x_{2} \geq \lambda_{5} \text { then } S \geq \lambda_{4} \\ \text { if } x_{1} \geq \lambda_{2} \text { and } x_{2} \geq \lambda_{3} \text { then } S \geq \lambda_{3}\end{array}\right\}$ where $\lambda_{2}<\lambda_{3}<$ $\lambda_{4}<\lambda_{5}$. Both rules involve the same criteria. Observe that the impossibility condition of Proposition 2 is not met. Due to the first rule, we must add a utility function $\varphi_{1}$ such that $\varphi_{1}\left(\lambda_{3}\right) \geq \lambda_{4}$ and $\varphi_{1}\left(x_{1}\right)<\lambda_{4}$ if $x_{1}<\lambda_{3}$. Due to the second rule, $\varphi_{1}$ must also satisfy $\varphi_{1}\left(\lambda_{2}\right) \geq \lambda_{3}$ and $\varphi_{1}\left(x_{1}\right)<\lambda_{3}$ if $x_{1}<\lambda_{2}$. For instance, one may choose $\varphi_{1}\left(\lambda_{1}\right)=\lambda_{2} ; \varphi_{1}\left(\lambda_{2}\right)=\lambda_{3} ; \varphi_{1}\left(\lambda_{3}\right)=\lambda_{4}$. Likewise for attribute 2, $\varphi_{2}$ must also satisfy $\varphi_{2}\left(\lambda_{5}\right) \geq \lambda_{4}$ and $\varphi_{2}\left(x_{2}\right)<\lambda_{4}$ if $x_{2}<\lambda_{5}$, and $\varphi_{2}\left(\lambda_{3}\right) \geq \lambda_{3}$ and $\varphi_{2}\left(x_{2}\right)<\lambda_{3}$ if $x_{1}<\lambda_{3}$, for instance $\varphi_{2}\left(\lambda_{2}\right)=\lambda_{2}, \varphi_{2}\left(\lambda_{3}\right)=\lambda_{3}, \varphi_{2}\left(\lambda_{4}\right)=\lambda_{3}, \varphi_{2}\left(\lambda_{5}\right)=\lambda_{4}$. Using utility functions we get single-thresholded rules

$$
\left\{\begin{array}{l}
\text { if } \varphi_{1}\left(x_{1}\right) \geq \lambda_{4} \text { and } \varphi_{2}\left(x_{2}\right) \geq \lambda_{4} \text { then } S \geq \lambda_{4} \\
\text { if } \varphi_{1}\left(x_{1}\right) \geq \lambda_{3} \text { and } \varphi_{2}\left(x_{2}\right) \geq \lambda_{3} \text { then } S \geq \lambda_{3} .
\end{array}\right.
$$

which can be represented by the single expression $S=\min \left(\varphi_{1}\left(x_{1}\right), \varphi_{2}\left(x_{2}\right), \lambda_{4}\right)$.

Here a question arises: what sort of rule sets can be represented with a Sugeno utility functional $S_{\mu, \varphi} ?$

The above results suggest that the set of rules must have a locally strict monotonic behavior, in the following sense: Let $R(j)$ be the set of rules where attribute $x_{j}$ appears. Let $\Theta_{j}$ be the set of thresholds $\alpha_{i}$ appearing in the rules $r^{i}$ of $R(j)$ in the form $x_{j} \geq \alpha_{i}$, and let $\Gamma(\alpha)$ be the set of conclusion thresholds $\delta_{i}$ for rules $r^{i}$ such that $\alpha_{i}=\alpha \in \Theta_{j}$. Then the multifunction $\Gamma$ must be strictly monotonic in the sense that $\forall \alpha, \alpha^{\prime} \in \Theta_{j}, \alpha>\alpha^{\prime}$ implies $\min \Gamma(\alpha)>\max \Gamma\left(\alpha^{\prime}\right)$. Indeed, note that if there are several conclusion thresholds $\delta \in \Gamma(\alpha)$, corresponding to several rules having the same condition threshold $x_{j} \geq \alpha$, the utility function for $x_{j}$ will have to satisfy $\varphi_{j}(\alpha) \geq \max \Gamma(\alpha)$ and $\varphi_{j}\left(x_{j}\right)<\min \Gamma(\alpha)$ if $x_{j}<\alpha$.

\section{Combination of Sugeno utility functionals}

In order to find an aggregation operation that can represent any set of multi-thresholded selection rules, we consider non decreasing functions $f: L^{n} \rightarrow L$ of the form

$$
f\left(x_{1}, \ldots x_{n}\right)=\max _{i \in I} \min \left(\min _{j \in A_{i}} \varphi_{i j}\left(x_{j}\right), \delta_{i}\right)
$$

where $L$ is a finite chain and each mapping $\varphi_{i, j}: L \rightarrow L$ is an increasing function such that $\varphi_{i, j}(0)=$ 0 and $\varphi_{i, j}(1)=1$. We call such functions $f$ piecewise unary functions in disjunctive form (df-PUF), in the sense that the domain $L^{n}$, can be partitioned into subsets where $f\left(x_{1}, \ldots x_{n}\right)=\varphi_{i j}\left(x_{j}\right)$ or is a constant $\delta_{i}$ for some $i \in C$.

The main purpose of this part is to study whether there exists a family of $K$ Sugeno utility functionals $S_{k}$ such that $f=\vee_{i=1}^{K} S_{k}$, and to show that any aggregation function $g$ (non-decreasing and such that $g(1,1, \ldots, 1)=1$ and $g(0,0, \ldots, 0)=0)$ can be expressed in this way. If this is so, we can then find a disjunction of Sugeno utility functionals that accounts for a set of selection rules, and more generally we can hope to learn such aggregation from qualitative data.

On the first issue we can prove the following result: 
Proposition 3. Any $d f-P U F$ on a finite domain such that $f(1,1, \ldots, 1)=1$ and $f(0,0, \ldots, 0)=0$ is a disjunction of Sugeno utility functionals.

Proof: First notice that as $f(1,1, \ldots, 1)=1$, there exists $i \in I$ such that $\delta_{i}=1$, and $\forall j \in$ $A_{i}, \varphi_{i j}(1)=1$; moreover $\forall j \in A_{i}, \varphi_{i j}(0)=0$. It is clear that we can rewrite $f$ as $f\left(x_{1}, \ldots, x_{n}\right)=$ $\left.\max _{i \in I} \max \left(\min \left(\min _{j \in A_{i}} \varphi_{i j}\left(x_{j}\right)\right), \delta_{i}\right), \min _{k \in C} \varphi_{i k}\left(x_{k}\right)\right)$, provided that $\forall k \notin A_{i}, \varphi_{i k}(1)=1$ and $\varphi_{i k}\left(x_{k}\right)=0$ if $x_{k}<1$. The inner expression

$$
\left.\max \left(\min \left(\min _{j \in A_{i}} \varphi_{i j}\left(x_{j}\right)\right), \delta_{i}\right), \min _{k \in C} \varphi_{i k}\left(x_{k}\right)\right)
$$

is a Sugeno utility functional with respect to the capacity $\mu_{i}$ such that $A_{i}$ is a focal set with $\mu_{i}\left(A_{i}\right)=\delta_{i}$ and $\mu_{i}(C)=1$ in the case $A_{i} \neq C$.

The decomposition of $f$ as $\bigvee_{i \in I} S_{\mu_{i}, \varphi_{i}}(x)$ in Proposition 3 is not parsimonious. Some terms inside $\bigvee_{i \in I}$ can be grouped into a single Sugeno utility functional with respect to a more complex capacity by unifying the utility functions for each attribute into a single one. To do so, the idea is that we extract a maximal number of subsets $A_{i} \subset C$, such that

- whenever $A_{i} \cap A_{i^{\prime}} \neq \emptyset$, the utility functions $\varphi_{i j}$ and $\varphi_{i^{\prime} j}$ for all $j \in A_{i} \cap A_{i^{\prime}}$ must be equal.

- whenever $A_{i} \subset A_{i^{\prime}}$, we have that $\delta_{i}<\delta_{i^{\prime}}$.

Let $I_{1}$ be the maximal subset of indices of terms that can form a Sugeno utility functional using the corresponding subsets of $A_{i}$ 's as described above. The idea is then to apply the same procedure to the remaining $\left\{A_{i}: i \in I \backslash I_{1}\right\}$, until no index remains in $I$.

Likewise we can consider piecewise unary functions in conjunctive form (cf-PUF), namely expressions such as:

$$
f\left(x_{1}, \ldots x_{n}\right)=\min _{i \in I} \max \left(\max _{j \in A_{i}} \psi_{i j}\left(x_{j}\right), \gamma_{i}\right) .
$$

Proposition 4. Any cf-PUF on a finite domain such that $f(1,1, \ldots, 1)=1$ and $f(0,0, \ldots, 0)=0$ is a conjunction of Sugeno utility functionals.

Proof: First notice that as $f(0,0, \ldots, 0)=0$, there exists $i \in I$ such that $\gamma_{i}=0$, and $\forall j \in$ $A_{i}, \psi_{i j}(0)=0$; moreover $\forall j \in A_{i}, \psi_{i j}(1)=1$. It is clear that we can rewrite $f$ as $f\left(x_{1}, \ldots, x_{n}\right)=$ $\min _{i \in I} \min \left(\max \left(\max _{j \in A_{i}} \psi_{i j}\left(x_{j}\right), \gamma_{i}\right), \max _{k \in C} \psi_{i k}\left(x_{k}\right)\right)$, provided that $\forall k \notin A_{i}, \psi_{i k}(0)=0$ and $\psi_{i k}\left(x_{k}\right)=1$ if $x_{k}>0$. The inner expression

$$
\min \left(\max \left(\max _{j \in A_{i}} \psi_{i j}\left(x_{j}\right), \gamma_{i}\right), \max _{k \in C} \psi_{i k}\left(x_{k}\right)\right)
$$

is a Sugeno utility functional (in conjunctive form) with respect to the capacity $\mu_{i}$ such that $A_{i}^{c}$ is a focal set with $\mu_{i}\left(A_{i}^{c}\right)=\gamma_{i}\left(\right.$ and $\left.\mu_{i}(\emptyset)=0\right)$.

Based on these results, we can try to model any aggregation function $g$ completely defined by a $n$-dimensional table, by means of a disjunction or a conjunction of Sugeno utility functionals. Indeed, we can represent such a table by means of a set of multi-thresholded selection or deletion rules. 
Example 4. Let us consider the function $f\left(x_{1}, x_{2}\right)$ in the table below where the scale is $0<\lambda<1$

\begin{tabular}{|c||c|c|c|}
\hline$x_{2} \uparrow x_{1} \rightarrow$ & 0 & $\lambda$ & 1 \\
\hline \hline 1 & 1 & 1 & 1 \\
\hline$\lambda$ & 0 & $\lambda$ & 1 \\
\hline 0 & 0 & 0 & $\lambda$ \\
\hline
\end{tabular}

We can describe the positive values in position $\left(\alpha_{1}, \alpha_{2}\right)$ in the table by means of selection rules of the form "if $x_{1} \geq \alpha_{1}$ and $x_{2} \geq \alpha_{2}$ alors $S \geq \delta$ ". In our example the following rules are enough:

\section{For output value 1}

$r^{1} x_{2}=1 \Rightarrow S=1$; (upper line)

$r^{2} x_{1}=1$ and $x_{2} \geq \lambda \Rightarrow S=1$ (value 1 in line 2 ).

\section{For output value $\lambda$}

$r^{3} x_{1} \geq \lambda$ and $x_{2} \geq \lambda \Rightarrow S \geq \lambda(\lambda$ in line 2$)$

$r^{4} x_{1}=1 \Rightarrow S \geq \lambda$ ( $\lambda$ in bottom line).

This set of rules can be expressed by means of a df-PUF formed by the maximum of the following terms

$-r^{1}: \varphi_{12}\left(x_{2}\right)$, with $\varphi_{12}(1)=1$ and $\varphi_{12}(\lambda)<1\left(\delta_{1}=1\right)$.

$-r^{2}: \min \left(\varphi_{21}\left(x_{1}\right), \varphi_{22}\left(x_{2}\right)\right)$ with $\varphi_{21}(1)=1$ and $\varphi_{21}(\lambda)<1 ; \varphi_{22}(\lambda)=1$ and $\varphi_{22}(0)=0\left(\delta_{2}=1\right)$.

$-r^{3}: \min \left(\varphi_{31}\left(x_{1}\right), \varphi_{32}\left(x_{2}\right), \lambda\right)$ with $\varphi_{31}(\lambda) \geq \lambda ; \varphi_{32}(\lambda) \geq \lambda\left(\delta_{3}=\lambda\right)$.

$-r^{4}: \min \left(\varphi_{41}\left(x_{1}\right), \lambda\right)$, with $\varphi_{41}(1)=1$ and $\varphi_{41}(\lambda)=0\left(\delta_{4}=\lambda\right)$.

By construction, the df-PUF that corresponds to the superposition of tables in Figure 1,

$$
\max \left(\varphi_{12}\left(x_{2}\right), \min \left(\varphi_{21}\left(x_{1}\right), \varphi_{22}\left(x_{2}\right)\right), \min \left(\varphi_{31}\left(x_{1}\right), \varphi_{32}\left(x_{2}\right), \lambda\right), \min \left(\varphi_{41}\left(x_{1}\right), \lambda\right)\right) \leq f\left(x_{1}, x_{2}\right),
$$

i.e., is a lower bound of function $f$.

\begin{tabular}{|c|c|c|}
\hline 1 & 1 & 1 \\
\hline$\geq 0$ & $\geq 0$ & $\geq 0$ \\
\hline 0 & 0 & 0 \\
\hline
\end{tabular}

\begin{tabular}{|l|c|c|}
\hline 0 & $\geq 0$ & 1 \\
\hline 0 & $\geq 0$ & 1 \\
\hline 0 & 0 & 0 \\
\hline
\end{tabular}

\begin{tabular}{|l|l|l|}
\hline 0 & $\lambda$ & $\lambda$ \\
\hline 0 & $\lambda$ & $\lambda$ \\
\hline 0 & 0 & 0 \\
\hline
\end{tabular}

$0|0| \lambda$

000

$00 \mid \lambda$

Fig. 1. $r^{1}: \varphi_{12}\left(x_{2}\right) \quad r^{2}: \min \left(\varphi_{21}\left(x_{1}\right), \varphi_{22}\left(x_{2}\right)\right) \quad r^{3}: \min \left(\varphi_{31}\left(x_{1}\right), \varphi_{32}\left(x_{2}\right), \lambda\right) \quad r^{4}: \min \left(\varphi_{41}\left(x_{1}\right), \lambda\right)$

It can be checked that the df-PUF acting as a lower bound of $f$ can be made equal to $f$ provided that $\varphi_{12}(\lambda)=0$.

Let us represent $f$ by the maximum of Sugeno utility functionals. First, rules $r^{1}$ and $r^{4}$ together correspond to the Sugeno utility functional $S_{\mu, \varphi}(x)$ where $\mu_{\#}(1)=\lambda, \mu_{\#}(2)=1$, where $\varphi_{1}(\lambda)=0=$ $\varphi_{2}(\lambda)$, i.e., we can choose $S_{\mu, \varphi}(x)=\left(\lambda \wedge \varphi_{1}\left(x_{1}\right)\right) \vee \varphi_{2}\left(x_{2}\right)$. Rule $r^{2}$ alone corresponds to the Sugeno utility functional $S_{\mu^{\prime}, \varphi^{\prime}}(x)$ where $\mu_{\#}(\{1,2\})=1$, and $\varphi_{2}^{\prime}(\lambda)=1$, that is $S_{\mu^{\prime}, \varphi^{\prime}}(x)=x_{1} \wedge \varphi_{2}^{\prime}\left(x_{2}\right)$. Note that the utility functions for rule $r^{3}$ can be chosen as being the same as those for rule $r^{2}$, 
in which case the term $\min \left(\varphi_{31}\left(x_{1}\right), \varphi_{32}\left(x_{2}\right), \lambda\right)$ is subsumed by $S_{\mu^{\prime}, \varphi^{\prime}}(x)$. It yields the following expression of $f$ :

$$
f\left(x_{1}, x_{2}\right)=S_{\mu, \varphi}(x) \vee S_{\mu^{\prime}, \varphi^{\prime}}(x)=\left(\lambda \wedge \varphi_{1}\left(x_{1}\right)\right) \vee \varphi_{2}\left(x_{2}\right) \vee\left(x_{1} \wedge \varphi_{2}^{\prime}\left(x_{2}\right)\right) .
$$

The values other than 1 in position $\left(\alpha_{1}, \alpha_{2}\right)$ in the table can also be represented by means of deletion rules of the form "if $x_{1} \leq \alpha_{1}$ and $x_{2} \leq \alpha_{2}$ then $S \leq \delta$ ". In our example the following rules are enough:

\section{For output value $\lambda$}

$$
\begin{aligned}
& r^{\prime 1} x_{2}=0 \Rightarrow S \leq \lambda ;(\text { value } \lambda \text { in line } 1) \\
& r^{\prime 2} x_{1} \leq \lambda \text { and } x_{2} \leq \lambda \Rightarrow S \leq \lambda(\text { value in line } 2) .
\end{aligned}
$$

For output value 0

$r^{\prime 3} x_{1}=0$ and $x_{2} \leq \lambda \Rightarrow S=0$ (value 0 in column 1 );

$r^{\prime 4} x_{1} \leq \lambda$ and $x_{2}=0 \Rightarrow S=0$ (value 0 in line 1 ).

This set of rules can be expressed by means of a piecewise unary function formed by the minimum of the following max-terms

$-r^{\prime 1}: \max \left(\psi_{12}\left(x_{2}\right), \lambda\right)$

$-r^{\prime 2}: \max \left(\psi_{21}\left(x_{1}\right), \psi_{22}\left(x_{2}\right), \lambda\right)$ with $\psi_{21}(\lambda) \leq \lambda ; \psi_{22}(\lambda) \leq \lambda$

$-r^{\prime 3}: \max \left(\psi_{31}\left(x_{1}\right), \psi_{32}\left(x_{2}\right)\right)$ with $\psi_{32}(\lambda)=0$

$-r^{\prime 4}: \max \left(\psi_{41}\left(x_{1}\right), \psi_{42}\left(x_{2}\right)\right)$ with $\psi_{41}(\lambda)=0$;

The piecewise unary function that corresponds to the superposition of tables in Figure 2 verifies $\min \left(\max \left(\psi_{12}\left(x_{2}\right), \lambda\right), \max \left(\psi_{21}\left(x_{1}\right), \psi_{22}\left(x_{2}\right), \lambda\right), \max \left(\psi_{31}\left(x_{1}\right), \psi_{32}\left(x_{2}\right)\right), \max \left(\psi_{41}\left(x_{1}\right), \psi_{42}\left(x_{2}\right)\right)\right) \geq f\left(x_{1}, x_{2}\right)$.

It can be checked that this piecewise unary function acting as an upper bound of $f$ becomes equal to $f$, provided that $\varphi_{12}(\lambda)=1$, which ensures value 1 on entry $(1, \lambda)$.

\begin{tabular}{|c|c|c|}
\hline 1 & 1 & 1 \\
\hline$\leq 1$ & $\leq 1$ & $\leq 1$ \\
\hline$\lambda$ & $\lambda$ & $\lambda$ \\
\hline
\end{tabular}

Fig. 2. $\quad r^{\prime 1}$

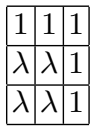

$r^{2}$

\begin{tabular}{|c|c|c|}
\hline 1 & 1 & 1 \\
\hline 0 & $\leq 1$ & 1 \\
\hline 0 & $\leq 1$ & 1 \\
\hline
\end{tabular}

$r^{\prime 3}$

\begin{tabular}{|c|c|c|}
\hline 1 & 1 & 1 \\
\hline$\leq 1$ & $\leq 1$ & 1 \\
\hline 0 & 0 & 1 \\
\hline
\end{tabular}

$r^{\prime 4}$

We can find utility functions in such a way that the upper bound coincides with the function $f$ and can be expressed as a minimum of Sugeno integrals. Namely rules $r^{\prime 1}$ and $r^{\prime 4}$ can be put together and yield a capacity $\mu$ such that $\mu(2)=\lambda$ and $\mu(\{1,2\})=1$. We can unify the utility functions appearing in the max-terms for these rules: $\psi_{1}(\lambda)=0, \psi_{2}(\lambda)=1$; rule $r^{\prime 3}$ yields a capacity $\nu$ such that $\nu(\{1,2\})=1$. We need utility function $\psi_{2}^{\prime}(\lambda)=0$, while $\psi_{1}^{\prime}$ can be the identity. Rule $r^{\prime} 2$ holds if we use the same utility functions as for rule $r^{\prime 3}$. We get:

$$
f\left(x_{1}, x_{2}\right)=\min \left(S_{\mu, \psi}(x), S_{\nu, \psi^{\prime}}(x)\right)=\min \left(\max \left(\lambda, \psi_{2}\left(x_{2}\right)\right), \max \left(\psi_{1}\left(x_{1}\right), \psi_{2}\left(x_{2}\right)\right), \max \left(x_{1}, \psi_{2}^{\prime}\left(x_{2}\right)\right)\right)
$$


To generalize the approach outlined in the above example we can consider the following steps.

1. Transforming the function into a set of selection (resp. deletion) rules.

2. Expressing each rule as a weighted min-term (resp. max-term) involving unary functions, and building the corresponding df-PUF (resp. cf-PUF).

3. Grouping min-terms (resp. max-terms) into Sugeno utility functionals, by unifying the utility functions for each involved variable.

In order to find the minimal set of selection rules that can represent (a lower bound of) an aggregation function $f$, we can proceed as follows. Consider $\delta=f\left(\lambda_{1}, \ldots, \lambda_{n}\right)$ and the Cartesian products of the form $\Lambda_{\delta}=\times_{i=1}^{n}\left[\lambda_{i}, 1\right]$. We can restrict to the maximal sets of this form in the sense of inclusion, i.e.,

$$
\mathcal{K}_{\delta}=\max _{\subseteq}\left\{\Lambda_{\delta}: \delta=f\left(\lambda_{1}, \ldots, \lambda_{n}\right),\left(\lambda_{1}, \ldots, \lambda_{n}\right) \in L^{n}\right\}
$$

For each such maximal hypercube $\Lambda_{\delta} \in \mathcal{K}_{\delta}$ and each $\delta>0$, we can write the selection rule

$$
\bigwedge_{i: \lambda_{i}>0} x_{i} \geq \lambda_{i} \Rightarrow f \geq \delta
$$

and construct a max-term following the procedure described earlier in this paper.

For deletion rules, the procedure is similar, but we consider maximal sets of the form $M_{\delta}=$ $\times_{i=1}^{n}\left[0, \lambda_{i}\right], \delta<1$ and for each of them, define the deletion rule

$$
\bigwedge_{i: \lambda_{i}<1} x_{i} \leq \lambda_{i} \Rightarrow f \leq \delta
$$

and construct a min-term.

While Step 2 of the above procedure is obvious to get a df-PUF (resp. cf-PUF), we again get a non-parsimonious representation. Moreover in order to have an exact representation of the aggregation function $f$ using selection rules only (or deletion rules only), we may need to enforce additional constraints on the utility functions as patent in Example 4. Finally, Step 3 should be more formally defined, as the choice of the groupings of max-terms (resp. min-terms) of the df-PUF (resp.cf-PUF), and the alignment of utility functions so as to form several Sugeno utility functionals to be combined does not seem to be unique. The question of finding a minimal representation of any aggregation function on a finite scale by means of conjunction or a disjunction of Sugeno utility functionals is a matter of further research.

It is interesting to measure the improved expressive power, when going from Sugeno integrals to monotonic aggregation functions on $L$. For instance in the case when $|L|=3$ as in Example 4, it is easy to check from Figure 3 that there are $49=7 \times 7$ idempotent aggregation functions, only 9 of which are Sugeno integrals, all of the form $\max \left(\min \left(x_{1}, \mu(1)\right), \min \left(x_{2}, \mu(2)\right), \min \left(x_{1}, x_{2}\right)\right)$, with $\mu(1), \mu(2) \in L$.

\section{Conclusion}

The main result of this paper is to show that any set of rules involving thresholds acting as lower bounds (resp. upper bounds) on attribute values or global evaluation can be represented by piecewise 


\begin{tabular}{|c|c|c|c|}
\hline$x_{2} \uparrow x_{1} \rightarrow$ & 0 & $\lambda$ & 1 \\
\hline 1 & $d \geq c$ & $\geq \max (\lambda, c)$ & 1 \\
\hline$\lambda$ & $c \leq \lambda$ & $\lambda$ & $\geq \max (\lambda, b)$ \\
\hline 0 & 0 & $a \leq \lambda$ & $b \geq a$ \\
\hline
\end{tabular}

Fig. 3. Aggregation functions on the three-valued scale

unary functions that in turn can be expressed in the form of fuzzy conjunctions or disjunctions of Sugeno integrals on suitable transformations of the common attribute scale. We have shown that this family of functions corresponds to monotonic aggregation functions on a finite scale. We have shown how to express such an aggregation function by means of a set of multi-thresholded rules, that in turn can be captured by combination of Sugeno utility functionals.

These results could be applied to learning aggregation operations (hence rules with thresholds) from qualitative data, viewing the latter as a partially defined aggregation table. There is another approach to this problem, based on Sugeno integrals and single-thresholded rules $[14,5]$. In these papers, the idea is to approach a set of data from above and from below by two standard Sugeno integrals with respect to an upper and a lower capacity, which is not always possible. In this method, there is no utility function. In contrast, an approach based on our result seems to lead to the conjunction or the disjunction of several Sugeno integrals (hence several capacities) and several unary functions acting as utility functions, which may require the tuning of many parameters. However, the latter drawback can be alleviated by searching for a minimal representation, which is a topic for further research.

Acknowledgements. This work is supported by ANR-11-LABX-0040-CIMI (Centre International de Mathématiques et d'Informatique) within the program ANR-11-IDEX-0002-02, project ISIPA.

\section{References}

1. M. Couceiro, D. Dubois, H. Prade and T. Waldhauser. Decision making with Sugeno integrals: DMU vs MCDM. Proc. 20th Eur. Conf. on Artificial Intelligence (ECAI 2012), IOS Press, 288-293, 2012.

2. M. Couceiro, D. Dubois, H. Prade and T. Waldhauser. Decision making with Sugeno integrals. Bridging the Gap between Multicriteria Evaluation and Decision under uncertainty. Order, 33 (3) 517-535, 2016.

3. M. Couceiro, J.-L. Marichal. Axiomatizations of quasi-polynomial functions on bounded chains. Aequationes Math. 396 (1), 195-213, 2009.

4. M. Couceiro, T. Waldhauser. Pseudo-polynomial functions over finite distributive lattices. Fuzzy Sets and Systems, 239, 21-34, 2014.

5. D. Dubois, C. Durrieu, H. Prade, A. Rico, Y. Ferro. Extracting decision rules from qualitative data using Sugeno integral: A case-study. Proc. ECSQARU'15, Springer, LNAI 9161, 14-24, 2015.

6. D. Dubois, J.-L. Marichal, H. Prade, M. Roubens, and R. Sabbadin. The use of the discrete Sugeno integral in decision making: A survey. Int. J. of Uncertainty, Fuzziness and Knowledge-Based Systems, 9:539-561, 2001.

7. D. Dubois, H. Prade. Qualitative possibility functions and integrals. In: Handbook of Measure Theory (E. Pap, ed.), Vol. 2, Elsevier, 1469-1521, 2002. 
8. D. Dubois, H. Prade, A. Rico. The logical encoding of Sugeno integrals. Fuzzy Sets and Systems, 241: 61-75, 2014.

9. D. Dubois, H. Prade, and R. Sabbadin. Decision-theoretic foundations of qualitative possibility theory. Eur. J. of Operational Research, 128:459-478, 2001.

10. M. Grabisch, T. Murofushi, and M. Sugeno (Eds.) Fuzzy Measures and Integrals. Theory and Applications. Physica-verlag, Berlin, 2000.

11. S. Greco, B. Matarazzo, R. Slowinski, Axiomatic characterization of a general utility function and its particular cases in terms of conjoint measurement and rough-set decision rules, Eur. J. Operarional Res. 158: 271-292, 2004.

12. J.-L. Marichal. Aggregation Operations for Multicriteria Decision Aid. Ph. D. Thesis, University of Liège, Belgium, 1998.

13. J.-L. Marichal. On Sugeno integrals as an aggregation function. Fuzzy Sets and Systems, 114(3):347-365, 2000 .

14. H. Prade, A. Rico, M. Serrurier, E. Raufaste. Elicitating Sugeno integrals: Methodology and a case study. Proc. ECSQARU'09, Springer, LNAI 5590, 712-723, 2009.

15. A. Rico. Modélisation des préférences pour l'aide à la décision par l'intégrale de Sugeno. PhD thesis, Université Paris I-Panthéon-Sorbonne, 2002.

16. A. Rico, C. Labreuche, M. Grabisch, and A. Chateauneuf. Preference modeling on totally ordered sets by the Sugeno integral. Discrete Applied Mathematics, 147, 2005.

17. M. Sugeno. Theory of Fuzzy Integrals and its Applications, Ph.D. Thesis, Tokyo Institute of Technology, Tokyo, 1974.

18. M. Sugeno. Fuzzy measures and fuzzy integrals: a survey. In: Fuzzy Automata and Decision Processes, (M. M. Gupta, G. N. Saridis, and B. R. Gaines, eds.), North-Holland, 89-102, 1977. 